\title{
Proposta de Framework para a Concepção de Ferramentas Voltadas a Domótica Assistiva de Baixo Custo
}

\author{
Gabriela D. Sousa ${ }^{1}$, Danilo Ferreira ${ }^{1}$, Cael Santos ${ }^{1}$, \\ Josivan R. Reis ${ }^{2}$, Roberto P. Nascimento ${ }^{1}$ \\ ${ }^{1}$ Instituto de Engenharia e Geociências - Universidade Federal do Oeste do Pará \\ (UFOPA) Santarém - PA - Brasil \\ ${ }^{2}$ Universidade Federal do Oeste do Pará (UFOPA) - Oriximiná - PA - Brasil \\ \{roberto.nascimento, josivan.reis\}@ufopa.edu.br \\ \{gabriela.dss, danilo.ferreira, cael.santos\}@discente.ufopa.edu.br
}

\begin{abstract}
With the technological advances of the last decades in the areas of robotics and Internet of Things, and with the aging of the population, the development of assistive systems has gained space in the residential environment. Thus, considering the costs and complexity involved in the construction and implementation of these products, the development of methodologies to assist in the planning and physical development of the product are of utmost importance. Therefore, this work presents a framework proposal to help in the development of projects in the assistive domotics field, aiming at low-cost products.
\end{abstract}

Resumo. Com o avanço tecnológico das últimas décadas em áreas do campo da robótica e da Internet das Coisas e com o envelhecimento populacional, o desenvolvimento de sistemas assistivos ganhou espaço no âmbito residencial. Assim, considerando os custos e complexidade envolvidos na construção e implementação desses produtos o desenvolvimento de metodologias para auxiliar no planejamento e no desenvolvimento físico do produto são de suma importância. Portanto, este trabalho apresenta uma proposta de framework para auxiliar no desenvolvimento de projetos no campo da domótica assistiva, visando produtos de baixo custo.

\section{Introdução}

Conforme os dados de [IBGE 2008] e [IBGE 2021], pessoas idosas, aquelas com 60 anos ou mais, representam $13 \%$ da população brasileira. Essa população tende a crescer nas próximas décadas e, em 2043, um quarto da população nacional deverá ser formada por idosos, segundo a Projeção da População do IBGE, atualizada em 2018. Segundo [World Health Organization (WHO) and The World Bank 2012], mais de um bilhão de pessoas no mundo são portadoras de deficiência, número que com o envelhecimento populacional apresenta uma tendência a crescer.

Segundo [Junior and Farinelli 2018], o termo domótica refere-se à realização de um controle automatizado de algo ou de algum ambiente utilizando-se da robótica. Sistemas domóticos buscam automatizar certos aspectos da residência de forma a proporcionar uma melhoria da qualidade de vida. O uso de dispositivos de domótica pode ser provar 
uma forma de providenciar, tanto a população idosa quanto as pessoas portadoras de deficiência, maior autonomia em suas residências, assim como é uma excelente alternativa para o monitoramento da residência através da presença de sensores instalados em diferentes cômodos, e para o monitoramento pessoal o qual visa auxiliar os moradores diante a um possível acidente, tal como quedas acidentais.

No entanto, o desenvolvimento de aplicações automotivas residenciais é um processo formado por um amplo conjunto de habilidades combinadas, as quais se direcionam a outros domínios específicos ligados, por instância, a questões ergonômicas, energéticas, sociais e tecnológicas, de forma que sua natureza se torna multidisciplinar e heterogênea, o que acaba por gerar uma elevada complexidade e demanda um longo período para ser concluída.

Neste trabalho será discutido sobre um framework voltado ao campo da domótica assistiva de baixo custo, através da divisão do processo de concepção e aprimoramento de produto em três etapas: Análise de Dados apresentada na Seção 3, Simulação apresentada na Seção 4 e Automação apresentada na Seção 5.

\section{Trabalho Relacionados}

Dada a complexidade no desenvolvimento de sistemas domóticos e das diversas especificações envolvidas nesse processo, tem-se que no trabalho de [Mishra et al. 2020] é apresentado um framework, o qual aborda uma estrutura genérica para a utilização da Realidade Aumentada (RA) e da Internet das Coisas (IoT) com o desenvolvimento de um sistema genérico e adequado a cada residência e as especificações de sistemas domóticos.

No estudo desenvolvido por [Gladence et al. 2020], é apresentado um framework para o desenvolvimento de sistemas de automação, com o objetivo de promover uma vida independente para a população idosa e portadora de deficiência, assim como, permite o monitoramento pessoal e residencial, e o controle e monitoramento do consumo de energia por equipamentos domésticos.

Em [BERNINI and SANTOS 2016] é proposto um ambiente inteligente de baixo custo para auxiliar pessoas de melhor idade e pessoas com deficiência, o trabalho utiliza como base as especificações minímas das residencias construídas pelo Programa Minha Casa Minha Vida. As automações são segmentadas em: equipamentos eletrodomésticos e eletrônicos, equipamentos de segurança e equipamentos de monitoramento de saúde, tais implementações podem ser usadas para prover qualidade de vida e conforto para pessoas com necessidades especiais e idosos de baixa renda.

\section{Análise de Dados}

A análise de dados é uma etapa fundamental para a criação de sistemas domóticos. Através de pesquisas literárias, revisão bibliográfica e da coleta de dados provenientes de outras ferramentas da automação, é possível conhecer trabalhos previamente realizados por outros estudiosos e gerar um programa de necessidades ou um banco de dados, por meio dos quais são verificadas as necessidades e características do usuário, do ambiente em que se deseja instalar a automação e dos sistemas já existentes, para assim, formular-se os requisitos funcionais do projeto o qual será desenvolvido e implementado. A Tabela 1 é uma exemplificação de um programa de necessidades, cujos questionamentos visam o desenvolvimento de produtos funcionais que atendam de forma adequada as 
necessidades de seus usuários e que possam ser inseridos no ambiente escolhido com o mínimo de reformas.

Tabela 1. Programa de necessidades

\begin{tabular}{|c|c|}
\hline Informações referentes & Questionamentos \\
\hline Ao usuário & $\begin{array}{l}\text { Qual o público-alvo? } \\
\text { Quais as principais características e necessidades } \\
\text { desse público-alvo? }\end{array}$ \\
\hline Ao ambiente & $\begin{array}{l}\text { Quais as medidas tridimensionais do ambiente? } \\
\text { Existe algum entrave para a instalação de dispositivos } \\
\text { de automação? }\end{array}$ \\
\hline A outros sistemas existentes & $\begin{array}{l}\text { Existem trabalhos e/ou estudos que se assemelham } \\
\text { com o produto que será desenvolvido? } \\
\text { Quais dificuldades e limitações foram encontradas no } \\
\text { decorrer do desenvolvimento e implementação desses } \\
\text { trabalhos? }\end{array}$ \\
\hline
\end{tabular}

Assim como, a partir de dados originários de sensores instalados em ambientes inteligentes, é possível utilizar de técnicas de Aprendizado de Máquina e Inteligência Artificial para o processamento e análise desses dados, de forma a otimizar o sistema e permitir uma maior adaptabilidade a diferentes cenários a qual o produto pode ser exposto.

Conforme a Figura 1, é observado que o processo de construção e aperfeiçoamento de um sistema domótico é tratado neste trabalho como um ciclo, no qual a Análise de Dados é responsável por proporcionar informações necessárias para o desenvolvimento da etapa de Simulação e por tratar e analisar feedbacks oriundos da etapa da Automação, como forma de melhorar o produto desenvolvido.

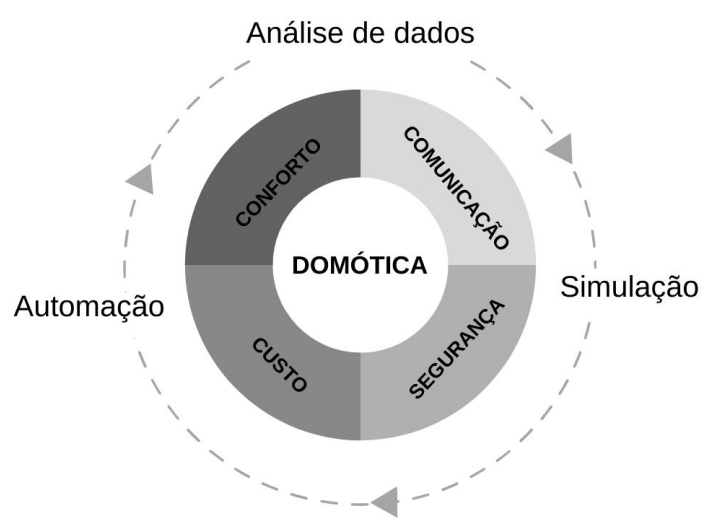

Figura 1. Framework Domótica Assistiva de Baixo Custo

\section{Simulação}

Considerando o programa de necessidades pode-se montar um ambiente de testes físico, porém, há um elevado custo na definição de sensores, instalação dos dispositivos e ajuste de configuração para preparar o ambiente de teste. A alternativa a esse processo seria 
montar uma simulação com base na análise dos dados. Um simulador permite que os custos relacionados a concepção do ambiente inteligente sejam drasticamente reduzidos, uma vez que todas as configurações necessárias serão realizadas em um ambiente virtual. Além disso, o uso de um simulador permite que o processo de coleta de dados de treino para inteligências artificiais se torne muito mais eficiente. Uma simulação pode gerar em questão de horas dados equivalentes a meses de observação assim como obter informações relacionadas a eventos impossíveis de serem reproduzidos em um ambiente real. Somado a isso, um ambiente virtual permite que se possa criar e testar sensores experimentais no ambiente simulado, facilitando o seu desenvolvimento.

No trabalho de [Synnott et al. 2015] é detalhado duas formas de aproximar simuladores para casas inteligentes: Model Based Approaches e Interactive Approaches. Model Based Approaches são simuladores onde o agente simulado segue um modelo de ações passado a ele pelo programa, este tipo de simulador é especialmente útil para gerar dados de treino, porém, a qualidade desses dados dependente da qualidade do modelo. Por outro lado, Interactive Approaches se referem aos simuladores onde o usuário tem controle total ou parcial sobre o agente da simulação. Enquanto Interactive Approaches não possuem a mesma capacidade de gerar grandes quantidades de dados de treino como Model Based Approaches, esse tipo de simulador é ideal para testar configurações de sensores com um maior grau de realismo. Deve-se considerar qual dessas abordagens será mais vantajosa na escolha ou desenvolvimento do simulador.

É preciso ressaltar que devido a natureza multidisciplinar do campo da Domótica temos que a facilidade de uso e de entendimento se tornam características de grande importância para um simulador. A capacidade de modificar o comportamento de um agente e de sensores sem ter que recorrer a edição de código ou edição de grandes arquivos de configuração permite que um grupo mais amplo de pesquisadores sejam capazes de criar ambientes que satisfaçam suas necessidades em menos tempo.

Com isso em mente, um ambiente de simulação foi construído durante o desenvolvimento deste framework. Tendo como base a plataforma de desenvolvimento de jogos Unity®, o simulador tem como foco uma Model Based Approach de fácil configuração, utilizando arquivos XML para definir tanto o modelo da rotina do agente simulado quanto as atividades que este pode realizar. O Unity ${ }^{\circledR}$ possibilita que sensores sejam facilmente distribuídos pelo ambiente 3D assim como permite que modelos criados em ferramentas externas sejam adicionados a simulação com facilidade.

\section{Automação}

Baseado no programa de necessidade e nos dados extraídos da simulação, é possível quantificar os sensores utilizados e quais dispositivos de automação poderão ser empregados no ambiente residencial. Com esta previa, é dada a oportunidade de procurar quais estruturas e plataformas que melhor se adéquam as necessidades do usuário, assim como é possível fazer uma pesquisa orçamentaria para os componentes necessários.

A automação neste projeto tem como base o uso de tecnologias assistivas. Para a construção de um ambiente acessível adequado, pode-se utilizar como base a norma ABNT NBR 9050/2020, esta norma propõe critérios e especificações técnicas para a construção de um ambiente acessível ideal, que promova segurança e independência à maior quantidade de pessoas, independentemente de idade, estatura ou limitação de mo- 
bilidade ou percepção [ABNT 2020].

Uma arquitetura de natureza genérica tem a vantagem de ser altamente personalizável, independente e suportar uma rede $I o T$ heterogênea, para alcançar tal objetivo, é necessário utilizar tecnologias baseadas em IoT e de plataforma open-source. Neste campo, se destacam as plataformas ESP8266 NodeMCU e Arduino ${ }^{1}$, ambas plataformas oferecem integração entre si e compatibilidade com grande parte dos sensores e módulos oferecidos pelo mercado.

O ESP8266 NodeMCU possui de forma nativa conexão via WIFI e interface que permite a integração por meio de portas digitais, analógicas, I2C, UART e PWM. O ESP8266 NodeMCU trabalhando em conjunto com o Arduino formam uma camada modular e configurável.

É preciso definir uma interface para a interação com o usuário, é interessante considerar interfaces de voz e via web. Soluções como Pocketsphinx ${ }^{2}$ e Vosk API $^{3}$ são opções viáveis para a construção de uma interface de voz robusta, ambas plataformas funcionam de forma offline e possuem suporte a vários idiomas, oferecendo a opção de treinar o próprio modelo de voz. A plataforma Vosk se destaca por possuir suporte para o Português e suporte para dispositivos leves, como Raspberry Pi, Android e iOS.

Seguindo o modelo proposto neste trabalho, ferramentas voltadas para automação residencial foram desenvolvidas. Destaca-se a construção de uma interface de voz, baseada no kit de ferramentas Vosk, que integra via rede WIFI outros artefatos, a interface se encontra instalada na plataforma Raspberry PI como observado na Figura 2. Outro destaque foi o desenvolvimento de um dispositivo para a automação de um ventilador de mesa, onde o dispositivo faz o controle da oscilação e da velocidade do ventilador por meio comandos de voz.

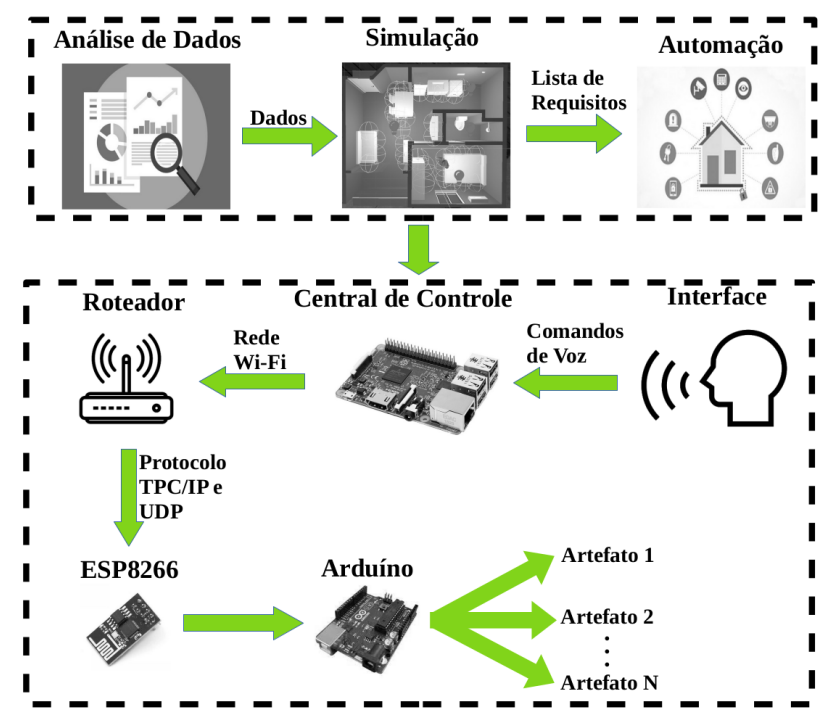

Figura 2. Estrutura da Automação

\footnotetext{
${ }^{1}$ https://www.arduino.cc/en/Guide/Introduction/

${ }^{2}$ https://pypi.org/project/pocketsphinx

${ }^{3}$ https://alphacephei.com/vosk/
} 


\section{Considerações finais}

Este trabalho teve como objetivo apresentar um framework voltado a criação e desenvolvimento de sistemas e produtos domóticos de baixo custo focados na área da saúde. Em seu decorrer foi introduzido a importância de se realizar pesquisas prévias, assim como as vantagens de se trabalhar com simulações antes de desenvolver o produto no mundo físico, além disso, foi apresentado uma estrutura para produzir automações residenciais.

Como trabalho futuro pretende-se refinar os projetos desenvolvidos durante a concepção desse framework com o objetivo de apresentar um protótipo de um ambiente domótico eficiente em relação a seus custos de implementação.

\section{Agradecimentos}

O presente projeto foi desenvolvido com apoio da Fundação Amazônia de Amparo a Estudos e Pesquisas - FAPESPA através do auxílio a projeto de pesquisa, $\mathrm{n}^{\circ}$ do processo 2019/585931, termo de outorga $\mathrm{n}^{\mathrm{o}} 006 / 2020$.

\section{Referências}

ABNT (2020). Associação Brasileira de Normas Técnicas - ABNT. NBR 9050/2020: Acessibilidade a edificações, mobiliário, espaços e equipamentos urbanos. Disponível em: http://www.museusacessiveis.com.br/arquivosDown/20201002100649_abnt-nbr9050-2020.pdf. Acessado em 02 de Maio de 2021.

BERNINI, R. d. A. and SANTOS, E. F. d. (2016). Habitações inteligentes para pessoas com deficiência e de melhor idade baseada no programa minha casa minha vida. $1^{o}$ Congresso Internacional de Ergonomia Aplicada.

Gladence, L. M., Anu, V. M., Rathna, R., and Brumancia, E. (2020). Recommender system for home automation using iot and artifcial intelligence. Journal of Ambient Intelligence and Humanized Computing.

IBGE (2008). Projeção da população do brasil e das unidades da federação. Disponível em: https://www.ibge.gov.br/apps/populacao/projecao/index.html. Acessado em 18 de Abril de 2021.

IBGE (2021). Idosos indicam caminhos para uma melhor idade. Disponível em: https://censo2021.ibge.gov.br/2012-agencia-de-noticias/noticias/24036-idososindicam-caminhos-para-uma-melhor-idade.html. Acessado em 20 de Abril de 2021.

Junior, S. L. S. and Farinelli, F. A. (2018). DOMÓTICA - Automação Residencial e Casas Inteligentes com Arduíno e ESP8266. Saraiva Educação S.A.

Mishra, A., Karmakar, S., Bose, A., and Dutta, A. (2020). Design and development of iot-based latency-optimized augmented reality framework in home automation and telemetry for smart lifestyle. Journal of Reliable Intelligent Environments.

Synnott, J., Nugent, C., and Jeffers, P. (2015). Simulation of smart home activity datasets. Sensors (Switzerland).

World Health Organization (WHO) and The World Bank (2012). Relatório Mundial Sobre Defiência. Governo do Estado de São Paulo, São Paulo. Tradução de Lexicus Serviços Lingüísticos. 